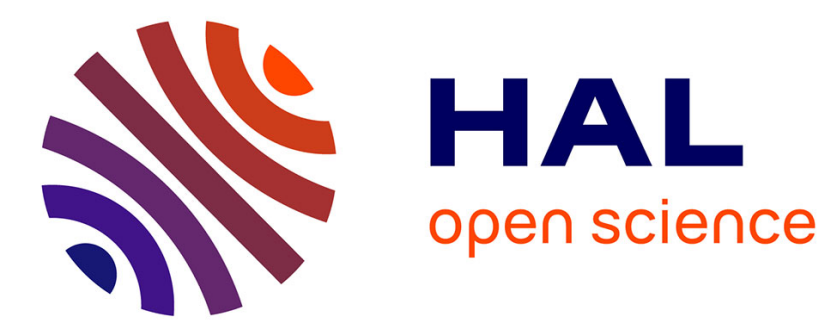

\title{
Les aqueducs de la villa de Vareilles à Paulhan (Hérault) : Ier s. av. J.-C.-IIIe s. apr. J.-C.
}

\author{
Stéphane Mauné, Jean-Louis Paillet, Zinedine Sekhari
}

\section{To cite this version:}

Stéphane Mauné, Jean-Louis Paillet, Zinedine Sekhari. Les aqueducs de la villa de Vareilles à Paulhan (Hérault) : Ier s. av. J.-C.-IIIe s. apr. J.-C.. Gallia - Archéologie de la France antique, 2005, Aqueducs de la Gaule méditerranéenne, 62, pp.131-145. 10.3406/galia.2005.3226 hal-01912657

\section{HAL Id: hal-01912657 https://hal.science/hal-01912657}

Submitted on 14 Jan 2020

HAL is a multi-disciplinary open access archive for the deposit and dissemination of scientific research documents, whether they are published or not. The documents may come from teaching and research institutions in France or abroad, or from public or private research centers.
L'archive ouverte pluridisciplinaire HAL, est destinée au dépôt et à la diffusion de documents scientifiques de niveau recherche, publiés ou non, émanant des établissements d'enseignement et de recherche français ou étrangers, des laboratoires publics ou privés.

\section{(ㅇ)(1) $\$$}

Distributed under a Creative Commons Attribution - NonCommercial - NoDerivatives $\mid 4.0$ 


\title{
LES AQUEDUCS DE LA VILLA DE VAREILLES À PAULHAN (HÉRAULT) : I $^{\mathrm{er}}$ S. AV. J.-C. - III S. APR. J.-C.
}

\author{
Stéphane MAUnÉ, Jean-Louis PAILLET et Zinedine SEKHARI
}

\begin{abstract}
Mots-clés. Gaule Narbonnaise, Haut-Lmpire, aqueducs ruraux, petite hydraulique, villa, économie domamiale.
Résumé. Fouillée sur près de 2,5 ha dans le cadre d'une opération préventive, la villa de Vareilles (Paulhan, Hérault) est occupée durant tout le Haut-Empire. Elle est dotée, dès l'époque augustéenne, d'un premier aqueduc vraisemblablement construit en grand appareil. Vers le milieu du $\mathrm{I}^{\mathrm{r}} \mathrm{s}$., un second aqueduc est construit tandis que le premier dispositif est remplacé, à la même période ou peu de temps après, par un ouvrage en béton de tuileau. Cette période voit aussi la mise en place d'un premier moulin hydraulique silué au point le plus bas de la villa. Dans les années 70 apr. J.-C., un balnéaire et de nouveaux bâtiments sont édifiés. La villa entre alors dans sa phase de splendeur: elle est alimentée par deux aqueducs et constitue un centre domanial de grande ampleur: Avec une capacité maximale de $8700 \mathrm{~m}^{3}$ d'eau par jour, les adductions d'eau assuraient un approvisionnement important et régulier. Les recherches menées sur ces ouvrages montrent toutefois qu'ils n'élaient pas exempts de défauts.
\end{abstract}

Key-words. Gallia Narbonensis, rural aqueducts, hydraulics, villa, estate economy, Principate.

Abstract. Being excavated on near 2,5 ha during a preventive operation, the villa of Vareilles (Paulhan, Hérault) was occupied all along the Principate, it was given as soon as the Augustan period a first aqueduct presumably built with masonry walls. Around the mid $1^{\text {rst }}$ century, a second one is built whereas the first installation is being replaced at the same time or soon after by a concrete work. A first water-mill also is built, situated at the lowest point of the villa. In the 1970s, a bath house and new buildings are added to the villa which enters a splendour phase, with water-supply coming from two aqueducts. It becomes a large scale country estate. Having a maximum capacity of $8700 \mathrm{~m}^{3}$ of daily water, the canalizations could provide a massive and regular supply. However they were not faultess as research on these installations has pointed out.

Translation : Isabelle FALDLET

Schlagwörter. Gallia Narbonnensis, Römische Wasserleitungen auf dem Land, Wasserbau im kleinen Maßstab, Villa, Domanialwirtschaft, frühe Kaiserzeit.

Zusammenfassung. Die im Rahmen einer Präventivgrabung auf einer Fläche von nahezu 2,5 ha freigelegte Villa von Vareilles (Paulhan, Dép. Hérault) war während der gesamten frühen Kaiserzeit besiedelt. Bereits von augusteischer Zeit an ist sie mil einer ersten Wasserleitung ausgerüstet, die wahrscheinlich von Anfang an in Mauerverkstechnik ausgeführt ist. Gegen Mitte des ersten Jahrhunderts n. Chr. wird eine zweite Leitung angelegt, während die ältere Einrichtung zur gleichen Zeit oier nur wenig später durch ein Bauwerk in opus caementitium ersetzt wird. In diese Zeit fällt auch die Errichtung einer ersten Wassermühle am tiefstgelegenen Geländepunkt der Villenanlage. Während der 70er Jahre werden ein Badegebäude und weitere Bauten errichtet. Damit bricht die Blütephase der Villa an. Durch zwei verschiedene. Wasserleitungen versorgt, bildet sie einen Domanialbetrieb von beträchtlicher (iröße. Die Leitungen garantierten mit einer Leistung von $8.700 \mathrm{~m}^{3}$ pro Tag für eine starke und regelmäßige Wasserzufuhr. Allerdings zeigen die Untersuchungen, daß diese Eïnrichtungen nicht immer einwandfrei funktionierten.

Überselzung : Stefan WIRTH

En Gaule Narbonnaise, et en particulier le long du littoral méditerranéen, la question de l'alimentation en eau devait être, notamment pour les grands propriétaires terriens, une préoccupation majeure, tant pour les besoins liés à la sphère domestique au sens large que pour les productions agricoles et artisanales. Face à cet enjeu majeur, les réponses apportées ont sans doute été diverses et variées, tant au niveau de l'approvisionnement qu'en ce qui concerne l'acheminement de l'eau. Malheureusement, nos connaissances restent encore modestes, en particulier pour la question des aqueducs ruraux qui nous 


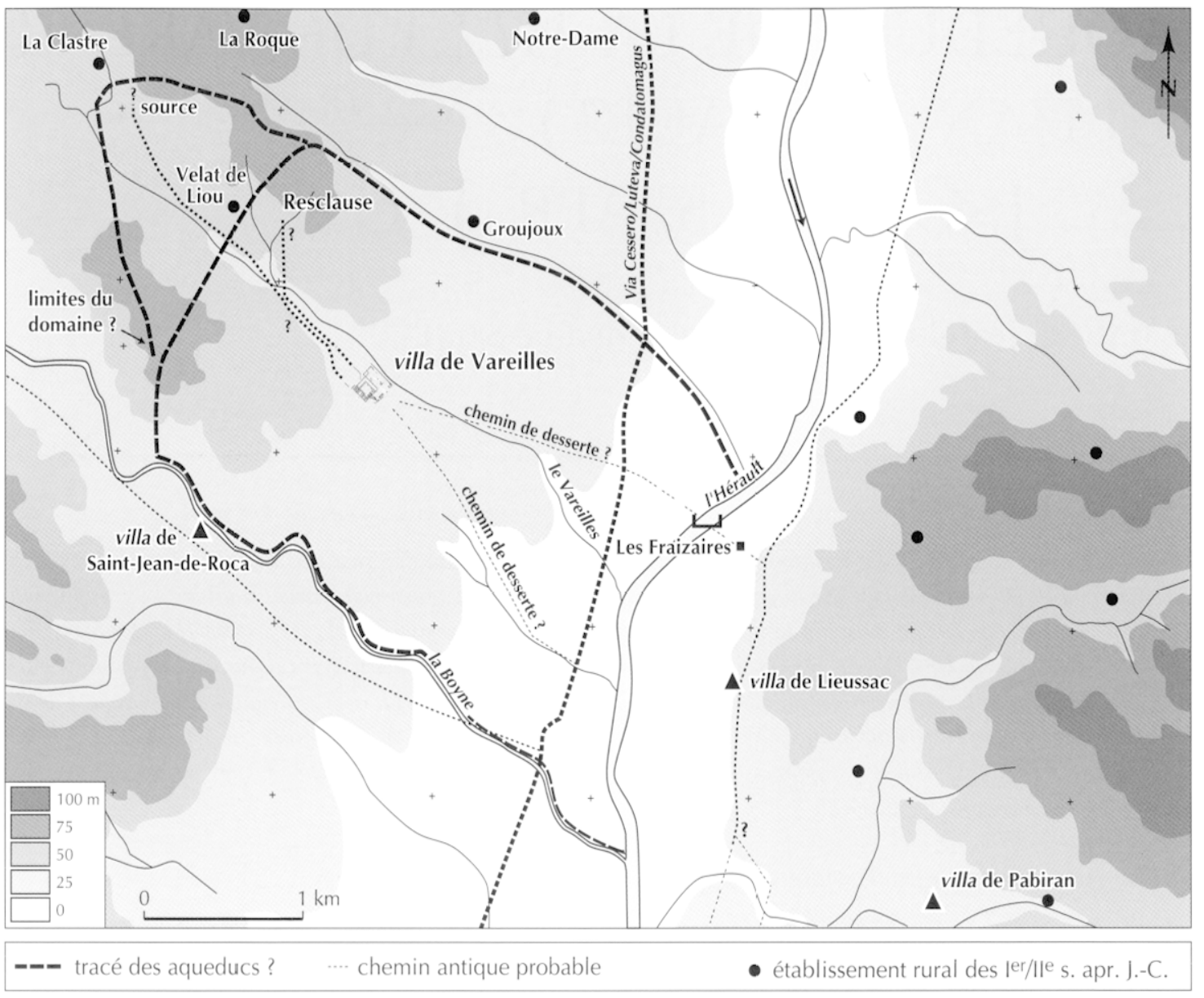

Fig. 121 - Localisation de la villa de Vareilles dans le contexte topographique local d'après les cartes IGN : $1 / 50000$ et villa : 1/25000 (DAOS. Mauné, CNRS).

occupe ici. Un rapide tour d'horizon des publications concernant les campagnes de Narbonnaise ne permet de réunir qu'un nombre assez limité d'exemples, inégalement documentés, et on ne dispose encore, à l'échelle provinciale, d'aucun inventaire exhaustif ${ }^{96}$. La plupart du temps, les découvertes et recherches ne concernent, sauf rares exceptions (par exemple Harfouche, Poupet, 2001), que des segments de faible ampleur ne permettant pas d'appréhender finement les relations entre ce type d'ouvrage et les établissements ruraux qu'il dessert. Enfin, bien peu d'exemples sont datés avec suffisamment de précision et la question de la construction et de

96. L'index, récemment publice, de la Revue archéologique de Narbonnaise permet par exemple d'observer que l'entrée "adduction d'eau " ne comporte qu'une mention ("Marseille, La Bourse ", RAN, 10, 1977, p. 240-245) concernant, qui plus est, une agglomération. La Carte archéologique du Var, publiée sous la direction de J-P. Brun et qui constitue actuellement la source documentaire la plus riche à notre disposition pour la Narbonnaise, comporte 17 mentions plus ou moins développées, relatives aux aqueducs desservant des établissements ruraux et/ou des villae (Brun dir., 1999). Quant aux deux volumes consacrés au Gard, nous n'avons pu collecter que quatre mentions d'aqueducs desservant une villa, dont une scule est indiscutable, la villa de Mayran à Saint-Victor-la-Coste, fouillée par L. Buffat. l'usage des aqueducs dans les derniers siècles de l'Antiquité reste posée.

Pourtant, il n'est que de constater la densité des grandes villae équipées d'installations thermales, dans certaines zones de la province (Bouet, 2003), pour se convaincre que le nombre des aqueducs ruraux en service devait être très élevé ${ }^{97}$. On soulignera aussi qu'en contexte domanial l'eau n'était pas, comme l'ont déjà prouvé certains travaux (Brun, Borréani, 1998), exclusivement réservée au secteur résidentiel, si bien qu'il faut estimer que les villae équipées d'un balnéaire n'étaient peut-être pas les seules à disposer de leur aqueduc.

On se propose d'aborder ici l'exemple de la villa de Vareilles dont la fouille extensive, conduite en 1999, a permis de mettre au jour deux aqueducs dégagés sur plusieurs centaines de mètres linéaires. L'ampleur des aménagements liés à la maîtrise de l'eau et à un approvisionnement régulier

97. Dans la région de Béviers, les recherches inédites de (;. Fedière ont ainsi permis de mettre en évidence l'existence d'une quinzaine d'aqueducs ruraux dont une présentation préliminaire a été faite à la table ronde: "L a petite hydraulique d'époque romaine dans les villue et établissements ruraux de Gaule Varbonnaise et des provinces voisines (I ${ }^{\mathrm{er}}$ s.-IT" s. apr. J.-(.) ", lemue à Pérenas (Hérault) les 22 et 23 mai 2003. 
confirme que la grande villa constitue un lieu privilégié pour l'étude des ourrages de petite hydraulique rurale.

\section{LA VILLA DE VAREILLES}

\section{PRÉSENTATION}

La villa de Vareilles se trouve à environ $35 \mathrm{~km}$ au nord-est de Béziers et à $25 \mathrm{~km}$ au sud-est de Lodève (voir supra fig. 1, p. 6). Elle est installée sur une terrasse alluviale ancienne, à une altitude d'environ $40 \mathrm{~m}$, au débouché d'un petit cours d'eau perpendiculaire au fleuve Hérault qui coule environ $2 \mathrm{~km}$ plus à l'est (fig. 121).

Le site a été découvert au début des années 1970 à l'occasion de travaux agricoles (Gallia, 33, 2, 1975, p. 508), puis a fait ensuite l'objet de prospections régulières (Mauné, 1998, p. 413-416) avant d'être fouillé en 1999 et 2001 dans le cadre d'une fouille préventive puis d'une opération programmée ${ }^{98}$.

\section{L'ÉVOLUTION DE LA VILLA ENTRE LE I ${ }^{\text {er }}$ S. AV. J.-C. ET LE III ${ }^{\mathrm{e}}$ S. APR. J.-C.}

Malgré l'état inégal de conservation stratigraphique et les dégâts causés par les travaux agricoles, l'évolution du site de Vareilles a pu être cernée dans ses grandes lignes, grâce notamment à l'abondance des mobiliers archéologiques (fig. 122).

\section{LA FONDATION DE L'ÉTABLISSEMENT (PHASE I)}

La phase I s'étend du tout début du I ${ }^{\mathrm{er}} \mathrm{s}$. av. J.-C. jusqu'aux années 40/30 av. J.-C. Elle voit l'installation, dans un vallon auparavant vide d'occupation, d'une ferme à enclos fossoyé couvrant environ $4000 \mathrm{~m}^{2}$.

\section{LA PREMIÈRE VILLA (PHASE II)}

Durant la phase II (30 av. J.-C./40 apr. J.-C.), des constructions, très mal conservées, sont installées sur l'emprise de la ferme d'époque tardo-républicaine. Elles se développent autour d'une cour ouverte au sud sur plus de $4000 \mathrm{~m}^{2}$. Á l'est, la fouille d'avril 2002 a révélé un bâtiment isolé qui pourrait marquer une extension des constructions dans cette zone. Plusieurs cuves en béton de tuileau ou en opus spicatum - dont la contemporanéité n'est pas certaine - attestent en tout cas la présence d'installations artisanales et/ou agricoles. L'existence d'un chai est rendue plausible par la mise au jour, aux portes

98. Dirigée par S. Mauné, la fouille AFAN/INRAP, intégrée au programme A75̃ placé sous la responsabilité de A. Chartrain (conservateur du Patrimoine, SRA Languedoc-Roussillon), s'est déroulée du

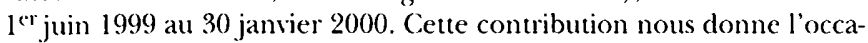
sion de remercier toute l'équipe de fouille qui s'est beaucoup investie dans la mise au jour de ces ourrages hydrauliques. Elle nous offre aussi l'opportunité de témoigner de notre attachement à une archéologie préventive en connexion avec les autres acteurs de la recherche, seule à même de renouveler en profondeur nos connaissances sur les campagnes gallo-romaines. mêmes de l'établissement, de trois parcelles de vigne dégagées sur plus de $8000 \mathrm{~m}^{2}$. Le site, désormais qualifié de villa en raison de la découverte d'éléments d'architecture de type résidentiel, disposait également d'un aqueduc, peut-être construit en grand appareil (roir infra), et dont le tracé, situé dans la partie basse du vallon, était peu ou prou parallèle à la rivière. La découverte, en contexte daté, de fragments de tubuli d'hypocauste laisse à penser que l'eau amenée par cet ourrage alimentait un petit balnéaire, mais ce seul argument reste néanmoins fragile.

\section{UNE GRANDE VLLA VITICOLE (PHASE III)}

Dans les années $40 / 50$ apr. J.-C. (phase III) est construit un très vaste édifice en $\mathrm{U}$ de $3600 \mathrm{~m}^{2}$ qui empiète largement sur des parcelles de vigne d'époque julio-claudienne. Ce vaste complexe de $60 \mathrm{~m}$ de côté semble presque entièrement voué à la viticulture. Il comporte un chai en $\mathrm{L}$ capable d'accueillir 350 dolia de grande taille. L'aqueduc augusto-tibérien (1 ${ }^{\text {er }}$ état, en grand appareil, voir infra) est abandonné ; son castellum est détruit et recouvert par le mur de façade nord de l'aile septentrionale du bâtiment à plan en U. Il n'est pas certain que l'aqueduc qui le recouvre et rejoint l'arrière de l'aile nordouest du complexe en U soit construit à cette époque. Peut-être n'apparaît-il que plus tard, lors de la phase IV. Quoi qu'il en soit, pour les installations vinicoles, un second aqueduc situé sur les terrasses supérieures est bâti. Les terrasses moyenne et basse sont désormais occupées par une cour et des installations artisanales. Un premier moulin à roue hydraulique verticale est construit. Il constitue l'aboutissement logique d'un système hydraulique complexe dont on ne perçoit malheureusement que quelques composantes. L'une des questions qui reste à élucider pour cette phase est celle de la localisation du secteur résidentiel: faut-il supposer qu'il se trouvait encore sur la terrasse moyenne malgré l'absence de vestige explicite? Peuton émettre l'hypothèse que la villa était alors dépourvue des appartements destinés au propriétaire et à sa familia? Il est difficile de répondre à ces questions à partir des seules données dont on dispose.

\section{UNE GRANDE VILLA DE NARBONNAISE (PHASE IV)}

La phase IV débute au tout début des années 70 apr. J.-C. : le complexe en $\mathrm{U}$ est fermé par une série de petites pièces sur lesquelles viennent s'appuyer, peu de temps après, des installations thermales. Ce dispositif résidentiel reste assez modeste mais la qualité des éléments de décoration qui ont pu y être exhumés - haut-relief représentant quatre pugilistes et un arbitre en toge ${ }^{99}$; élément de fontaine ou de nymphée comportant une frise de poissons - tranche avec ce que l'on connaît dans d'autres villae contemporaines de Narbonnaise. À l'arrière de ce dispositif, un bâtiment rectangulaire disposant d'une galerie de circulation est installé et rient fermer une cour, limitée sur son quatrième côté par un verger. À la même époque, un deuxième moulin est construit à proximité

99. L'étude de cet élément décoratif a été assurée par J.-P. Thuillier (ENS) et sera publiée dans la monographie (Mauné dir., à paraître). 

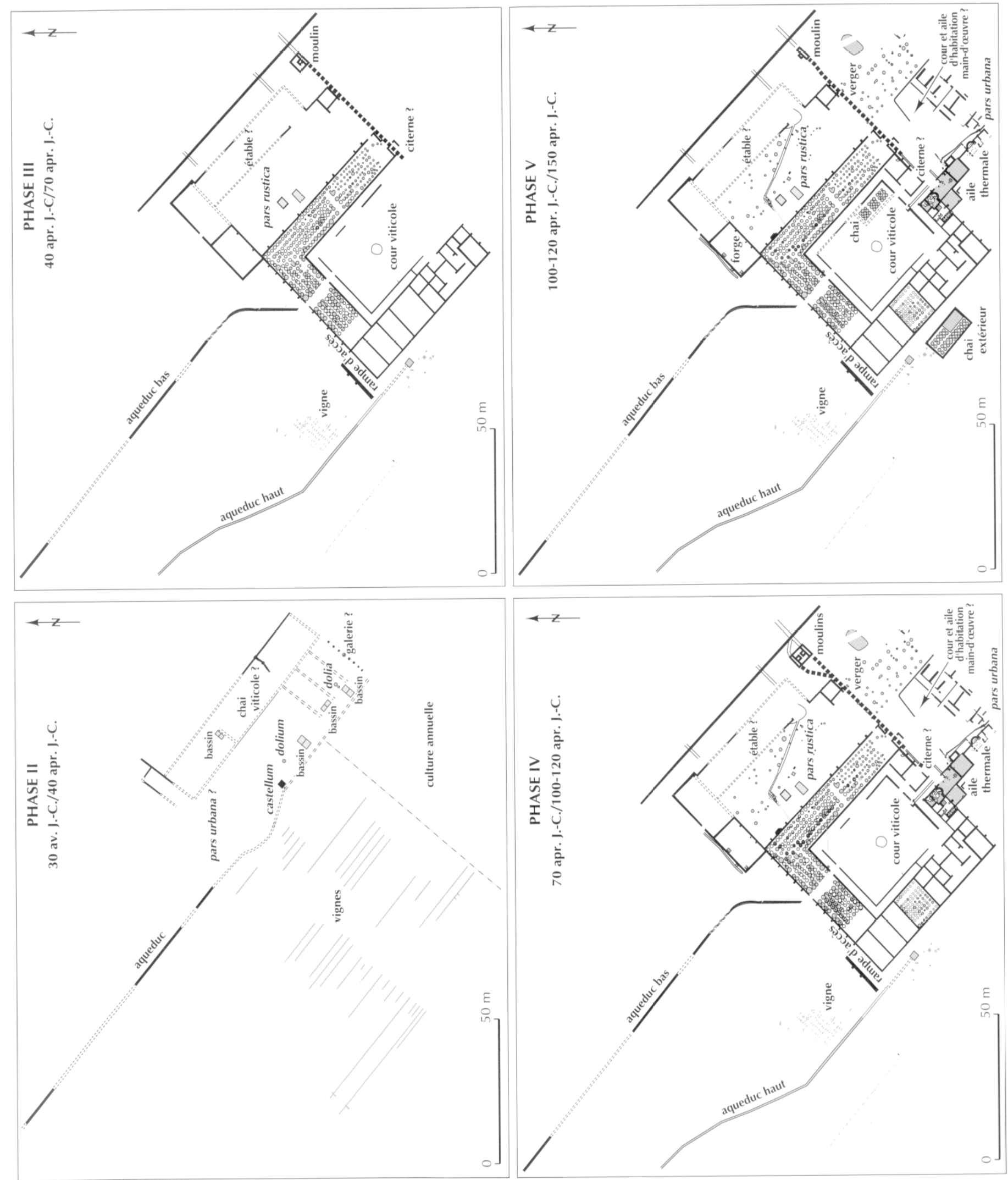

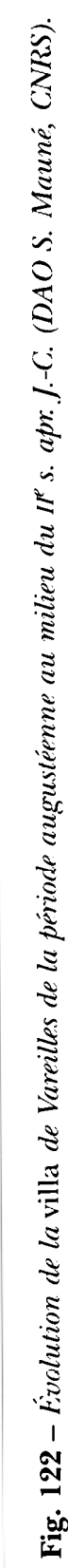


immédiate du précédent; enfin, un deuxième chai d'environ 70 dolia est implanté non loin du grand chai en $\mathrm{L}$, dans le complexe en U d'époque claudienne. C'est au plus tard à cette époque que l'on édifie, sur le tracé de l'ancien aqueduc augusto-tibérien, un second ouvrage, cette fois-ci en béton de tuileau, qui alimente un réservoir situé à l'arrière du complexe vinicole. C'est peut-être aussi durant cette phase que le canal de l'ouvrage haut est rehaussé.

\section{L'APOGÉE (PHASE V)}

La phase V est datée du début du $\mathrm{II}^{\mathrm{e}} \mathrm{s}$. Elle voit l'augmentation des capacités productives de la villa puisque deux nouveaux chais de petite taille sont construits. Un troisième moulin, de plus grande dimension, est bâti et remplace les unités précédentes. Dans la pars urbana, les thermes font l'objet de transformations diverses. Cette phase correspond à l'extension maximale de la villa puisque cours et constructions couvrent désormais plus de $12000 \mathrm{~m}^{2}$.

\section{LE DÉCLIN ET L'ABANDON (PHASE VI)}

La phase VI, enfin, marque le déclin progressif de la villa: à partir des années 150 apr. J.-C. et tout au long de la seconde moitié du II ${ }^{\mathrm{e}} \mathrm{s}$. Les chais sont abandonnés un à un, les égouts sont progressivement comblés, des dépotoirs sont installés dans les mares et dépressions diverses. Vers 220 apr. J.-C. au plus tard, la villa semble définitivement abandonnée et ne connaît aucune réoccupation ultérieure.

\section{LES AQUEDUCS}

Situés immédiatement à l'amont des bâtiments de la villa, ils ont été dégagés et fouillés de façon exhaustive sur plusieurs centaines de mètres linéaires (fig. 123). Dès la mise au jour de ces ouvrages, l'équipe de fouille a sollicité le concours d'un spécialiste, J.-L. Paillet, qui a participé à la fouille et fourni indications et conseils.

\section{LES AQUEDUCS BAS}

Nous disons "les " aqueducs bas parce que nous montrerons plus loin que sur un même tracé se sont succédé chronologiquement deux aqueducs différents. Les aqueducs bas ont été reconnus sur une longueur de $230 \mathrm{~m}$ environ et se développent dans trois zones de fouilles différentes (zones 2,5 et 3 en partant de l'amont vers l'aval). Le tracé de ces aqueducs n'est pas linéaire mais présente quatre segments d'orientation différente. La raison de ces changements de direction est liée à la microtopographie du bassin versant.

L'état de conservation des vestiges de ces ouvrages n'est pas uniforme. Selon les parcelles dans lesquelles se trouvent les différents segments, les destructions et spoliations postantiques ont affecté les vestiges antiques de manière plus ou moins importante. Par souci de clarté nous présenterons ensemble toutes les informations relatives à l'aqueduc primitif dans les différents segments.

\section{LE PREMIER AQLEDUC BAS}

Le segment $\mathrm{n}^{\circ} \mathrm{l}$, le plus en amont, se développe sur unc longueur de $50 \mathrm{~m}$. À cet endroit les restiges maçonnés du second aqueduc bas, le plus récent des deux, n'existent plus. N'en subsistent que quelques éléments en fond de tranchée de spoliation (altitude 40,18 m) (fig. 124). Cette tranchée d'épierrement est emboîtée dans une autre plus profonde et plus ancienne. Ce constat a pu être corroboré par d'autres observations effectuées sur plusieurs sections relevées sur les trois principaux segments de l'ouvrage. Ce premier aqueduc n'a été repéré que par sa tranchée fantôme, ou par sa semelle de fondation, constituée par un hérisson de pierres de calcaire molassique posées, soit sur le fond de la tranchée, soit insérées dans une couche de terre de nivellement rapportée après le creusement de la tranchée (altitude $39,90 \mathrm{~m}$ ) (coupe 2S15). Les deux coupes transversales relevées sur ce segment témoignent de ces deux cas de figure. Une troisième indique une légère variation de tracé de $0,70 \mathrm{~m}$ (2S13).

Le deuxième segment du tracé de cet aqueduc se développe sur une longueur de $30 \mathrm{~m}$ et marque un angle de $10^{\circ}$ vers l'ouest par rapport à sa direction initiale (2S11 et 2P20).

Ici, les vestiges du premier aqueduc sont apparus directement après le décapage d'une très épaisse couche arable, sans qu'aucun vestige de l'aqueduc le plus récent ne soit mis au jour. Ainsi, aucune tranchée de récupération similaire à celle observée dans la coupe 2S14 n'a pu être repérée (2S11, US 2355). Pour expliquer cette particularité, on propose l'hypothèse suivante : au moment de la spoliation de l'aqueduc le plus récent, la couche arable avait une épaisseur inférieure à celle d'aujourd'hui (1,10 m environ) ; la tranchée d'ćpierrement a donc été détruite par les travaux agricoles avant que de nouveaux apports de terre viennent recouvrir totalement le secteur.

En définitive, les seuls vestiges visibles correspondent à ceux de l'ouvrage le plus ancien. Il s'agit, d'une part, de sa tranchée de fondation (FO 2376), de son comblement inférieur (US 2375) et, d'autre part, d'un hérisson qui constituait sa semelle (SB 2365). Au-dessus de cette dernière, une couche limono-argileuse jaunâtre (US 2355) doit être interprétée comme le comblement naturel de sa tranchée de récupération.

Lors de la fouille, l'attention a été attirée par la parfaite horizontalité de la surface du hérisson de cette semelle (SB 2365, 2366, 2367). Le caractère organisé de cette structure doit également être souligné. En effet, elle est composée d'éclats de taille de moellons et de rebuts de diverses dimensions $(8 \mathrm{~cm}$ à $50 \mathrm{~cm})$ soigneusement agencés et posés sur un lit de terre étalée dans le fond de la tranchée de fondation initiale. Certains blocs portaient des traces d'outils caractéristiques (marteau taillant, pic, etc.).

L'emprise au sol de cette semelle varie entre $0,88 \mathrm{~m}$ et $0,60 \mathrm{~m}$, soit environ 3 et 2 pieds antiques. Compte tenu, d'une part, de l'extrême régularité du lit d'attente formé par le hérisson et, d'autre part, de l'absence totale de déchets de mortier de chaux dans les remblais de la spoliation, nous sommes conduits à supposer l'existence d'un aqueduc non pas maçonné mais construit en blocs de grand appareil taillés en $\mathrm{U}$, aboutés et jointoyés selon la technique des « joints à cordon 


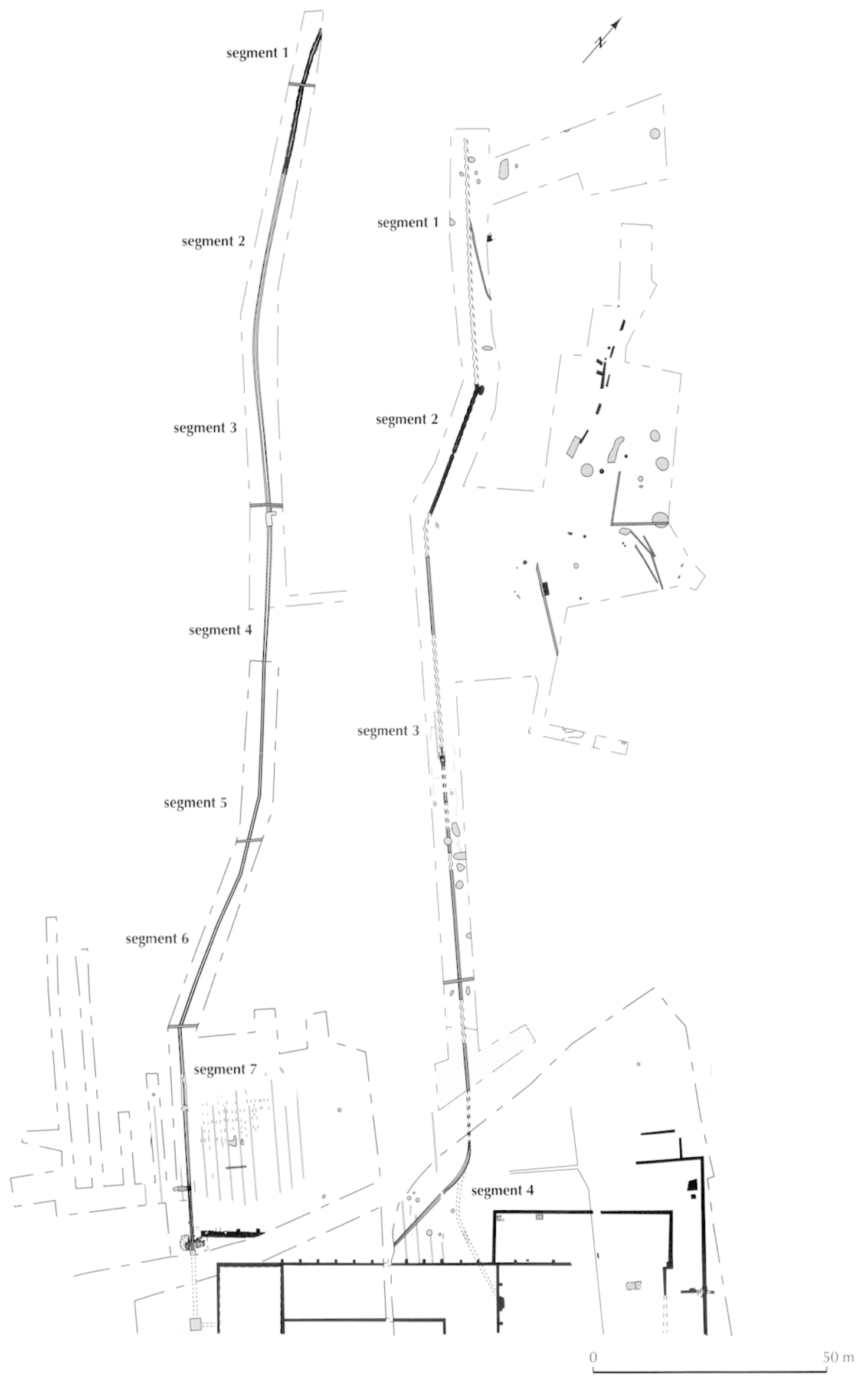

Fig. 123 - Plan du tracé des aqueducs en amont de la villa de Vareilles et la localisation des segments étudiés (I)AOS. Mauné, (NRS el I: Rébière, AFAN). 
prisonnier ". L'absence de tout remploi de ce type de bloc dans la villa post-claudienne de Vareilles nous oblige à supposer que ces blocs de grand appareil ont été débités et réutilisés dans les élévations des murs qui ont été à leur tour récupérés.

Le troisième segment, parfaitement rectiligne, mesure $125 \mathrm{~m}$ environ. Les vestiges du premier aqueduc sont moins bien conservés que dans le segment $\mathrm{n}^{\circ} 2$, ce dont témoignent les différentes coupes relevées. Pour expliquer cette particularité nous devons supposer que la semelle de fondation a été récupérée en même temps que les blocs de grand appareil. La trace de ce premier aqueduc n'est visible que par sa tranchée dont le profil supérieur devait être évasé ${ }^{100}$. Elle est épierrée et toujours située en dessous de l'ouvrage qui lui a succédé.

Dans le quatrième segment qui, à l'approche de la villa, oblique brusquement vers le sud-ouest, nous n'avons retrouvé aucune trace de l'aqueduc primitif. Les seuls vestiges conservés appartiennent indéniablement à l'aqueduc maçonné au mortier de chaux. Lors de la fouille de la villa, un sondage profond, implanté sur le mur de façade nord-ouest du grand chai en L, a montré la présence insoupçonnée d'un creusement linéaire antique dont la direction correspond à celle du segment $n^{\circ} 3$ du premier aqueduc. Force est de constater qu'entre ce sondage et le segment $n^{\circ} 3$ existe apparemment une solution de continuité. Comme le montre la coupe du creusement, une couche de substrat remanié (galets et graviers enrobés d'argile), indétectable en surface, a recouvert le fantôme de sa tranchée de récupération. En conséquence, nous devons accepter que le tracé de l'aqueduc primitif se poursuivait de façon rectiligne jusqu'à l'établissement qui a précédé la grande villa à cour centrale, et dont on restitue justement la localisation dans la zone 5 (terrasse moyenne).

Certes, cet aqueduc desservait la villa primitive, mais son aboutissement a un castellum nous reste inconnu. Toutes les traces de ce monument, qui est indispensable dans le fonctionnement et la distribution de l'eau, ont été oblitérées par la construction du grand chai de la villa rurale. Ne subsiste de ce dispositif qu'une fosse de spoliation située sous le mur de façade nord de l'aile septentrionale.

En fonction des informations recueillies dans les trois segments, quelles dimensions peut-on proposer pour les blocs en grand appareil creusés en L' qui constituaient cet aqueduc? La largeur maximale de l'assise constituée par les cailloux de molasse de la semelle est de l'ordre de 3 pieds. Aussi, nous proposons une largeur de bloc de 2 pieds pour une hauteur de 2 pieds. En supposant que l'épaisseur des parois était de l'ordre de $1 / 2$ pied, cela nous conduit à estimer la section mouillée de cet aqueduc à un pied carré, soit $0,087 \mathrm{~m}^{2}$, si l'on présume que le canal était rempli aux deux tiers.

La différence de niveau du fond de la tranchée entre les deux points extrêmes conservés (39,60 m et 39,12 m) est de $0,48 \mathrm{~m}$ pour une distance de $186 \mathrm{~m}$ et donc de $25,8 \mathrm{~cm}$ pour $100 \mathrm{~m}$.

100. La profondeur de la tranchée imposait un évasement de ses parois. De plus, sa partie inférieure adoptait un profil en cuvette. Cette cuvette a dû être nivelée par une couche de terre plus ou moins importante, puis recouverte par un hérisson destiné à assurer une assise stable d'au moins 3 pieds de large.

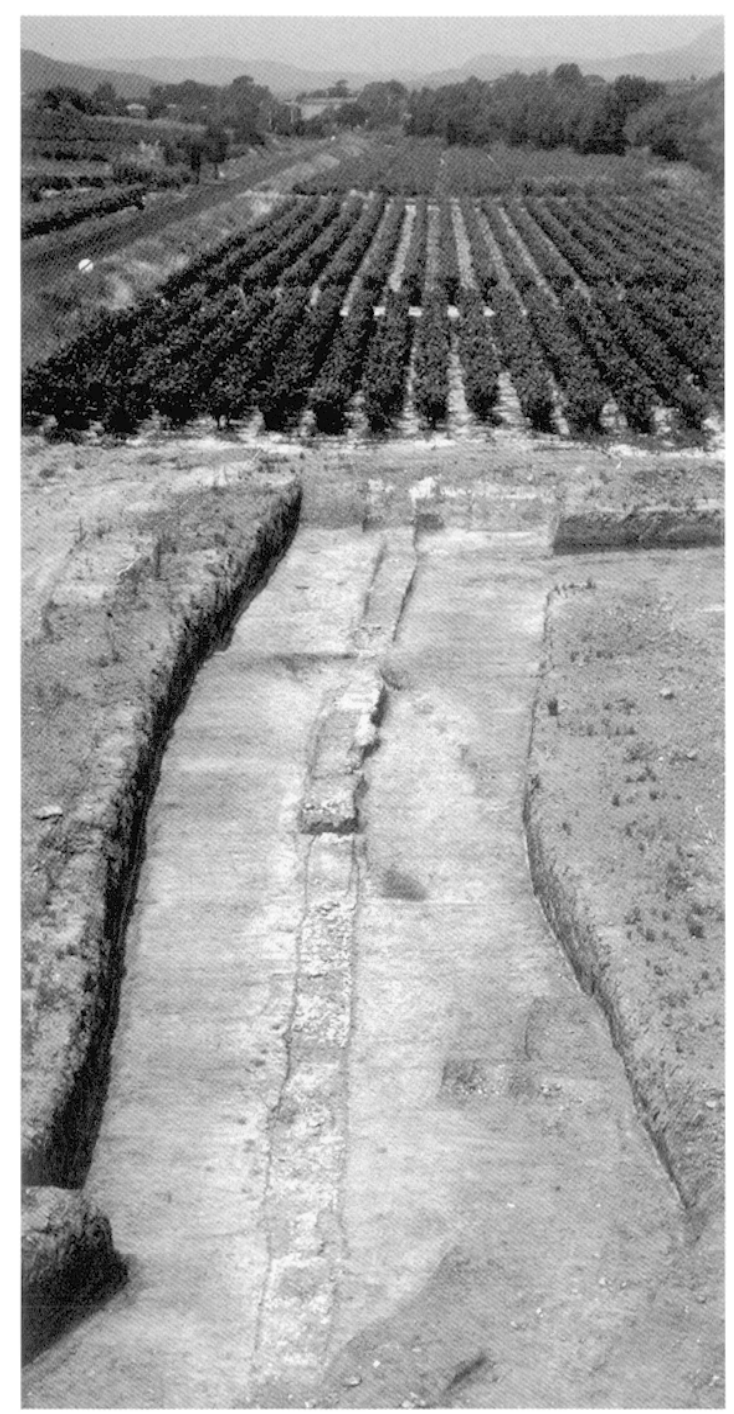

Fig. 124 - Villa de Vareilles : vue du segment 1 et de la fondation du premier aqueduc bas en direction de l'amont (cliché S. Mauné, CNRS).

À partir de la formule de Bazin et des données qui précèdent, il est possible de calculer le débit théorique de ce premier aqueduc : $0,0759 \mathrm{~m}^{3} / \mathrm{s}$.

Le débit potentiel estimé de ce premier aqueduc est donc de $76 \mathrm{l} / \mathrm{s}$ soit $6562 \mathrm{~m}^{3} /$ jour. Il est important de rappeler que, pour parvenir à ce résultat, on n'a que la largeur moyenne du lit d'attente des blocs disparus formé par la semelle empierrée de leur fondation. Ce résultat est donc une évaluation à considérer avec toutes les précautions d'usage, qui permet de réfléchir à partir d'un ordre de grandeur. Il montre que la villa de la phase II avait à sa disposition une importante quantité d'eau journalière et donc probablement des installations d'une certaine ampleur.

La chronologie de ce premier aqueduc bas ne peut pas être précisée dans l'absolu. Les seules informations susceptibles d'être prises en compte pour caler une chronologie relative sont de deux ordres. On peut tout d'abord souligner que les techniques de construction qui ont été employées lors de sa 


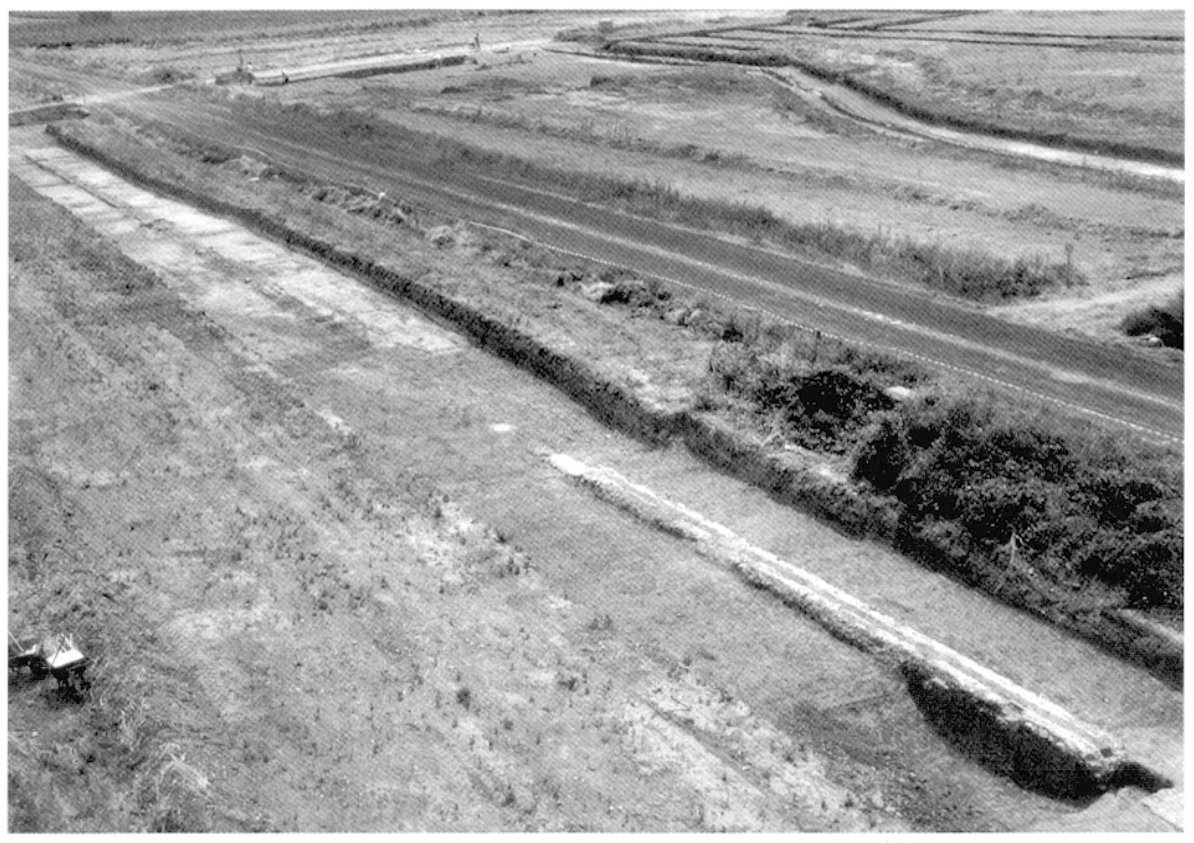

Fig. 125 - Villa de Vareilles : vue du segment 3 de l'aqueduc bas en cours de fouille (cliché S. Mauné, CNRS).
Dans le segment $\mathrm{n}^{\circ} 3$, l'aqueduc est conservé de façon inégale (fig. 125). Sur les $125 \mathrm{~m}$ de son développement, il n'est conservé que sur $62 \mathrm{~m}$ en six tronçons distincts et de longueur très variable $(16 \mathrm{~m}, 3 \mathrm{~m}, 1 \mathrm{~m}, 5 \mathrm{~m}, 27 \mathrm{~m}$ et $10 \mathrm{~m})$. Sur ces $62 \mathrm{~m}$, son état de conservation est relativement homogène ; les murs bajoyers de la canalisation sont conservés sur une dizaine de centimètres de hauteur, mais nulle part n'ont été retrouvés, ni la partie supérieure des murettes, ni son système de couverture. Pour décrire cet aqueduc, nous disposons du plan général, de plusieurs coupes et d'une série de photographies.

Sa technique de construction est semblable dans les différents tronçons (fig. 126). Dans le fond d'une tranchée ouverte à l'aplomb de la tranchée de spoliation de l'aqueduc de l'état précédent, a été déposée, en guise de hérisson, une couche de galets roulés de 10 à $20 \mathrm{~cm}$ de diamètre, irrégulièrement posés à plat ou de chant et noyés dans un limon argileux de couleur brun-gris homogène et relativement dense. Par la suite, un coffrage filant, en bois, de forme trapézoïdale et légèrement évasé vers le haut, a été mis en place sur des cales soigneusement nivelées. Un béton de tuileau a ensuite été coulé à l'extérieur du coffrage, par-dessous et de part et d'autre de ses parois. Après la prise de cette maçonnerie, le coffrage a été retiré pour être positionné sur les cales suivantes au fur et à mesure de la progression linéaire du chantier. À l'extrémité aval du deuxième tronçon conservé, l'ouvrage présente un élargisscment carré de $74 \mathrm{~cm}$ de côté, interprété comme une cuve dc décantation (fig. 127).

À l'extrémité aval de ce troisième segment, l'altitude NGF du fond du canal est de 39,72 m. Cela nous permet d'estimer la pente de cet aqueduc entre les deux points extrêmes du chantier où des cotes altimétriques ont pu être relevées à $68 \mathrm{~cm}$ pour un développé de $184 \mathrm{~m}$, soit $36,9 \mathrm{~cm}$ pour $100 \mathrm{~m} \mathrm{ou}$, approximativement, $0,4 \%$. Il faut toutefois remarquer que cette pente moyenne, relativement élevée, n'est pas uniforme selon les segments. 


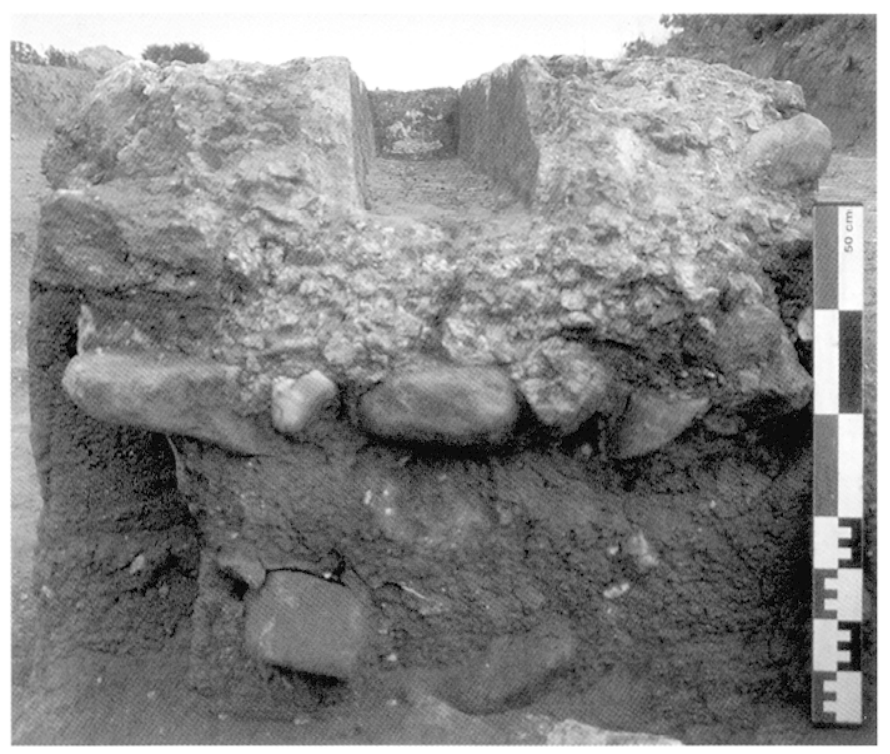

Fig. 126 - Villa de Vareilles : coupe du second aqueduc bas dans le segment 3 avec sa fondation en galets liés à l'argile (cliché S. Mauné, (NRS).

À l'extrémité du segment $n^{\circ} 3$, l'aqueduc n'a pas pu être observé car il était recouvert sur une dizaine de mètres par l'emprise d'un chemin rural goudronné. Au-delà, nous sommes dans la zone 5 de la villa. La tranchée de spoliation de l'aqueduc (segment $n^{\circ} 4$ ) marque un net changement de direction vers le sud et a pu être reconnue sur environ $30 \mathrm{~m}$. Rappelons à ce propos que le tracé de l'aqueduc primitif, d'époque augustéenne, conserve son tracé linéaire.

Les contraintes de sécurité liées à la déviation de la route départementale n'ont pas permis d'appréhender les vestiges de l'aqueduc à son entrée dans la villa, ni de son éventuel castellum. Lne hypothèse peut cependant être formulée. Le bassin devait être adossé au grand mur à contrefort limitant la villa au nordouest. Au-delà de cet ouvrage, une ou plusieurs conduites traversaient le chai et pouvaient desservir soit par la galerie, soit par la cour, les différents points de distribution. Cette hypothèse de restitution est argumentée par la découverte d'un tuyau en plomb d'axe nord-sud à proximité immédiate de la galerie nord-ouest.

Les données rassemblées lors de la fouille de cet ouvrage nous ont permis de restituer la physionomie du canal dans lequel l'eau circulait : celui-ci présentait une section d'environ 1 pied carré (environ $30 \mathrm{~cm} \times 30 \mathrm{~cm}$ ) semblable à celle de son précédent.

À partir des chiffres dont on dispose, notamment la pente fixée à $4 \%$, et toujours selon la formule de Bazin, le débit théorique de ce second ourrage est de : $0,0293 \mathrm{~m}^{3} / \mathrm{s}$, soit $29 \mathrm{l} / \mathrm{s}$ et $2531 \mathrm{~m}^{3} /$ jour.

\section{DATATION}

Nous ne disposons, pour dater cet aqueduc, d'aucun élément de chronologie autre que l'examen du plan géné-

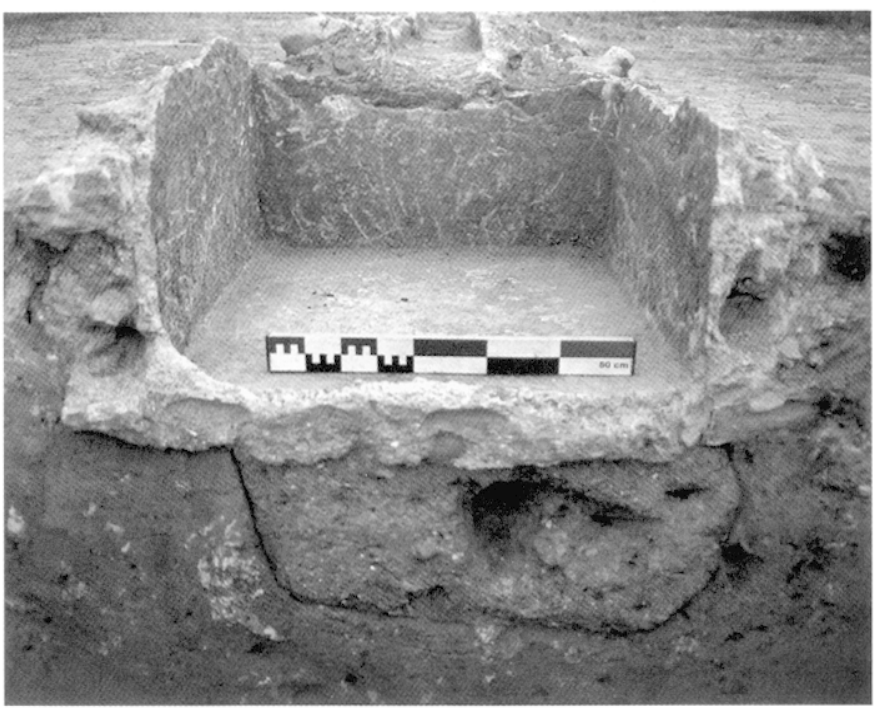

Fig. 127 - Villa de Vareilles : vue en coupe de la cuve de décantation du second aqueduc bas. On distingue, sous la cuve, le comblement de la tranchée de spoliation du premier ouvrage augustéen (cliché S. Mauné, CNRS).

ral des vestiges et la mise en perspective de la position des bâtiments et des structures linéaires tels les égouts par exemple.

Jusqu'à très récemment il semblait que le second aqueduc bas pouvait être rattaché à la phase III et qu'il était contemporain de la construction du grand bâtiment en U. Dans les années 40/60 apr. J.-C., deux aqueducs auraient alimenté l'établissement. Nous préférons aujourd'hui être plus prudents et élargir cette proposition à la phase IV. En effet, ne serait-il pas plus logique de mettre cet aqueduc en relation avec le développement des bâtiments vers l'est et la construction d'une aile thermale? Cette solution expliquerait la position peu " orthodoxe " de la partie terminale de cet aqueduc par rapport à un bâtiment l'ayant en fait précédé dans le temps ${ }^{102}$. Cependant, rien ne nous assure de la validité de cette hypothèse, aussi préférons-nous laisser l'interprétation ouverte.

\section{L'AQUEDUC HAUT}

L'aqueduc haut a été observé sur une longueur totale continue de $263,50 \mathrm{~m}$ (fig. 128). On peut toutefois lui restituer une longueur supplémentaire de $14 \mathrm{~m}$ dans son parcours terminal, juste avant une large fosse pouvant correspondre à la spoliation de son castellum. Cette conduite se développe parallèlement à l'aqueduc bas, mais sur une courbe de niveau située à une altitude supérieure de $1 \mathrm{~m}$. Comme pour l'ourrage bas,

102. Le point d'arrivée de l'aqueduc contre le grand mur à contreforts de l'aile nord-ouest du complexe vinicole n'a pu être fouillé en raison de la présence d'une bande de terrain non accessible bordant la déviation de la route départementale établie par la DDE pour nous permettre de fouiller la bordure nord de la cour centrale. Cette bande apparaît sur la figure 122 sous la forme d'un blanc dans le chai en I. 


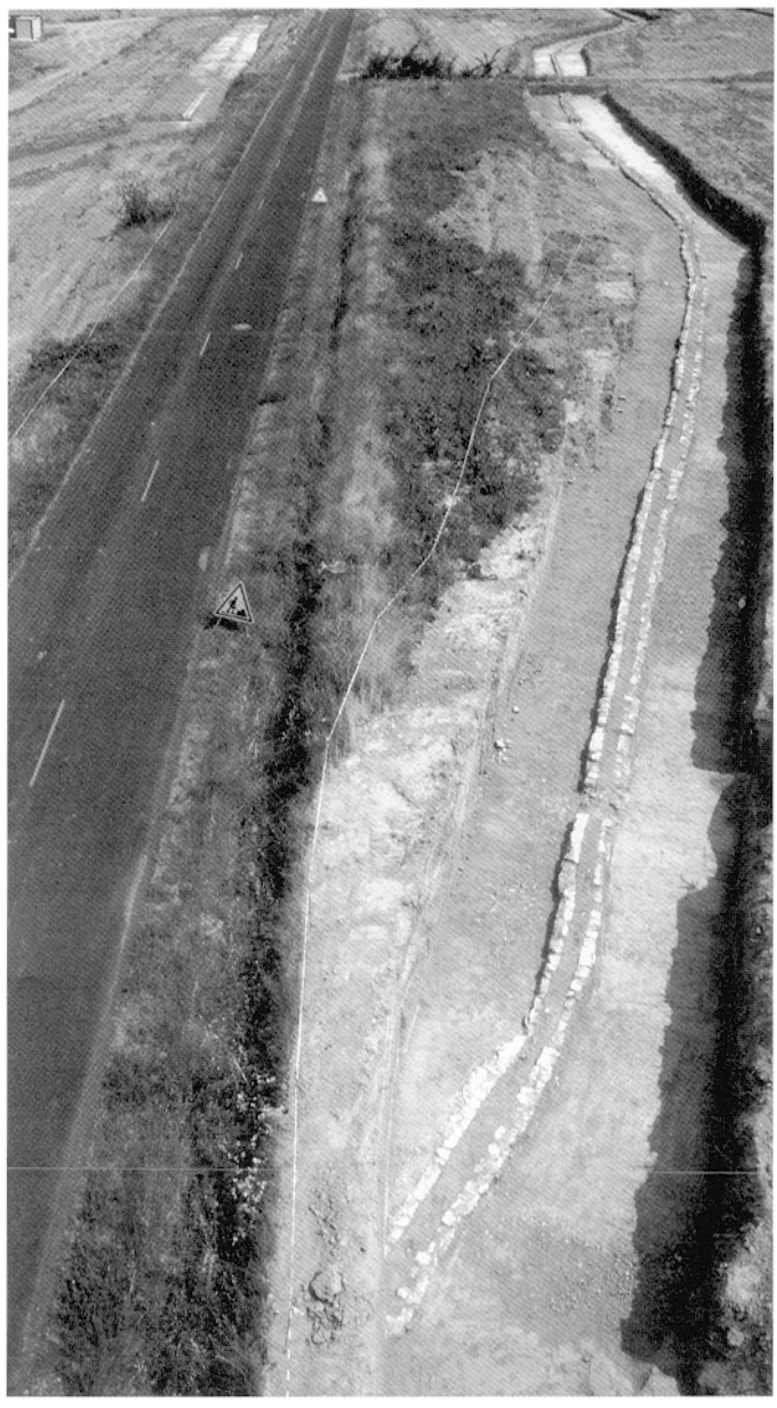

Fig. 128 - Villa de Vareilles : vue de l'amont de l'aqueduc haut (cliché S. Mauné, CNRS).

son tracé à été découpé selon les changements de direction en sept segments.

La configuration de ses six premiers segments indique que le concepteur a dû tenir compte de la présence d'un léger relief qu'il a fallu contourner afin de réduire la profondeur de la tranchée de construction et donc le coût des terrassements. En revanche, le dernier segment observé s'insère dans le parcellaire augustéen préexistant occupé par de la vigne. Il est d'ailleurs parallèle au tracé de l'aqueduc bas (état ancien) qui limitait ce parcellaire au nord.

Contrairement à l'aqueduc bas, l'aqueduc haut est bien conservé. Son élévation est visible sur la quasi-totalité de son tracé, et trois dalles de couverture, en place, étaient encore présentes dans le segment 7 terminal. Partout ailleurs, ce dispositif ainsi que les traces de son éventuelle spoliation n'ont pas été observés. Cela suscite un certain nombre de questions. Nous y reviendrons dans l'interprétation de la vocation précise de cet ouvrage.

\section{DESCRIPTION PAR SEGMENT}

Le segment 1 correspond au secteur fouillé le plus en amont (fig. 129). Sa longueur dégagée est de 7,50 m mais elle devait être plus importante dans la mesure où l'on devine, au-delà, en amont, son prolongement. C'est ici que le recouvrement sédimentaire est le plus important $(1,70 \mathrm{~m})$.

Le segment 2 se développe sur une longueur de $54,50 \mathrm{~m}$ et présente les mêmes caractéristiques que le segment 1 . Toutefois, l'épaisseur du recouvrement sédimentaire diminue sensiblement du nord vers le sud. Il est intéressant de constater que les changements d'orientation entre les segments 1, 2, 3 et 4 s'opèrent suivant une courbe très douce.

Le segment 3 s'étire sur $41 \mathrm{~m}$ de long et ne présente aucune différence avec les deux segments précédents. L'épaisseur du recouvrement sédimentaire continue de décroître.

Le segment 4 se poursuit sur 55,50 m, mais on doit signaler qu'il n'a été observé que sur $44 \mathrm{~m}$. Les $11,50 \mathrm{~m}$ restants correspondent à une zone traversée par un chemin agricole en service, bordé par deux fossés.

Le segment 5 se développe sur 16,50 $\mathrm{m}$ à l'extrémité orientale d'un petit éperon géologique qui, nous le verrons plus tard, constitue aussi un point haut de la canalisation. C'est également à partir de ce segment que les changements de direction de la canalisation sont opérés suivant des angles sans aucun amortissement. Cela laisse supposer des contraintes liées à la présence d'aménagements agraires établis à la périphérie immédiate de la villa.

Le segment 6 mesure $33,50 \mathrm{~m}$. Il a été épierré en partie, mais sa structure est semblable à celle des segments précédents.

Le segment 7 (fig. 130), très rectiligne, a $59 \mathrm{~m}$ de long et se terminait sur une grande fosse de spoliation supposée être le castellum. Toutefois, l'aqueduc n'a été dégagé que sur $44 \mathrm{~m}$. Dans sa partie terminale, la disparition des vestiges peut s'expliquer par deux facteurs distincts. C'est, tout d'abord, la présence d'un chemin creux médiéval (de Nizas à Paulhan) qui a fait disparaitre la canalisation sur environ $6 \mathrm{~m}$. D'autre part, aux abords immédiats des bâtiments de la villa (zone 1 ), on a pu observer la faiblesse de l'épaisseur sédimentaire et l'affleurement des vestiges antiques. Il y a donc eu manifestement, un remodelage de la microtopographie du secteur et sur une surface très restreinte, une érosion considérable des sols.

Sur le flanc nord-ouest de la villa, la fouille a mis en évidence une fosse rectangulaire d'environ $0,80 \mathrm{~m}$ de profondeur qui, se trouvant parfaitement dans le prolongement de l'aqueduc, nous a semblé pouvoir être interprétée comme le négatif du castellum aquae.

\section{OBSERVATIONS TECHNIQUES SUR CET AQUEDUC ET INTERPRÉTATION}

Une première observation s'impose; cet aqueduc est curieusement bâti (fig. 131 et 132). De toute évidence, les vestiges en place témoignent de deux états successifs qui sont probablement très proches l'un de l'autre. Le premier état ayant fait l'objet d'une erreur de nivellement, la plus grande partie de l'aqueduc a fait l'objet d'une surélévation immédiatement postérieure à la construction initiale. 


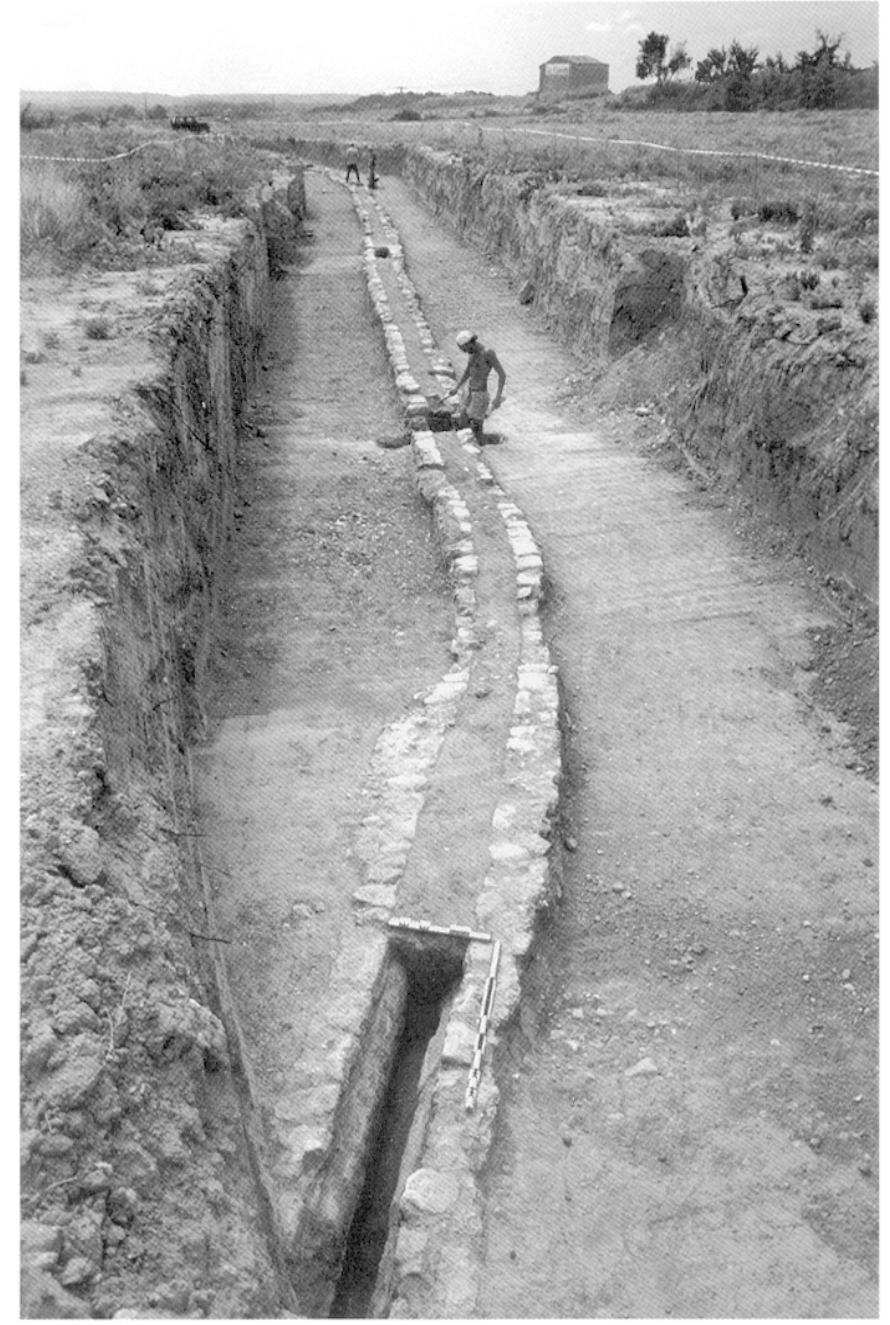

Fig. 129 - Villa de Vareilles : vue de l'amont du segment 1 de l'aqueduc haut en cours de fouille (cliché S. Mauné, CNRS).

Après l'installation d'une tranchée, large d'environ $0,75 \mathrm{~m}$, un hérisson constitué de galets roulés liés par du mortier de chaux a été déposé au fond. Un coffrage filant en bois, légèrement évasé, semble avoir été mis en place dans la partie centrale de la tranchée et les parois de la canalisation ont été respectivement coulées entre l'une des deux banches du coffrage et le bord extérieur de la tranchée qui, de fait, servait de coffrage perdu. Les parois de la canalisation sont donc en béton banché de chaux de couleur blanche et leur épaisseur movenne est de l'ordre de $0,25 \mathrm{~m}$, tandis que leur hauteur varie de $0,25 \mathrm{~m}$ à $0,28 \mathrm{~m}$. Le sommet de ces murs banchés correspond approximativement à celui d'un épandage de cailloutis compacté d'époque würmienne provenant probablement du creusement de la tranchée de construction de l'aqueduc. Il s'agit donc du niveau de circulation contemporain du premier état de l'ouvrage. Probablement au même moment, un cuvelage d'étanchéité en mortier de tuileau fin a été appliqué sur le fond, les parois latérales et sur une partie de la face supérieure des murs bajoyers de la conduite. C'est sur le fond de la

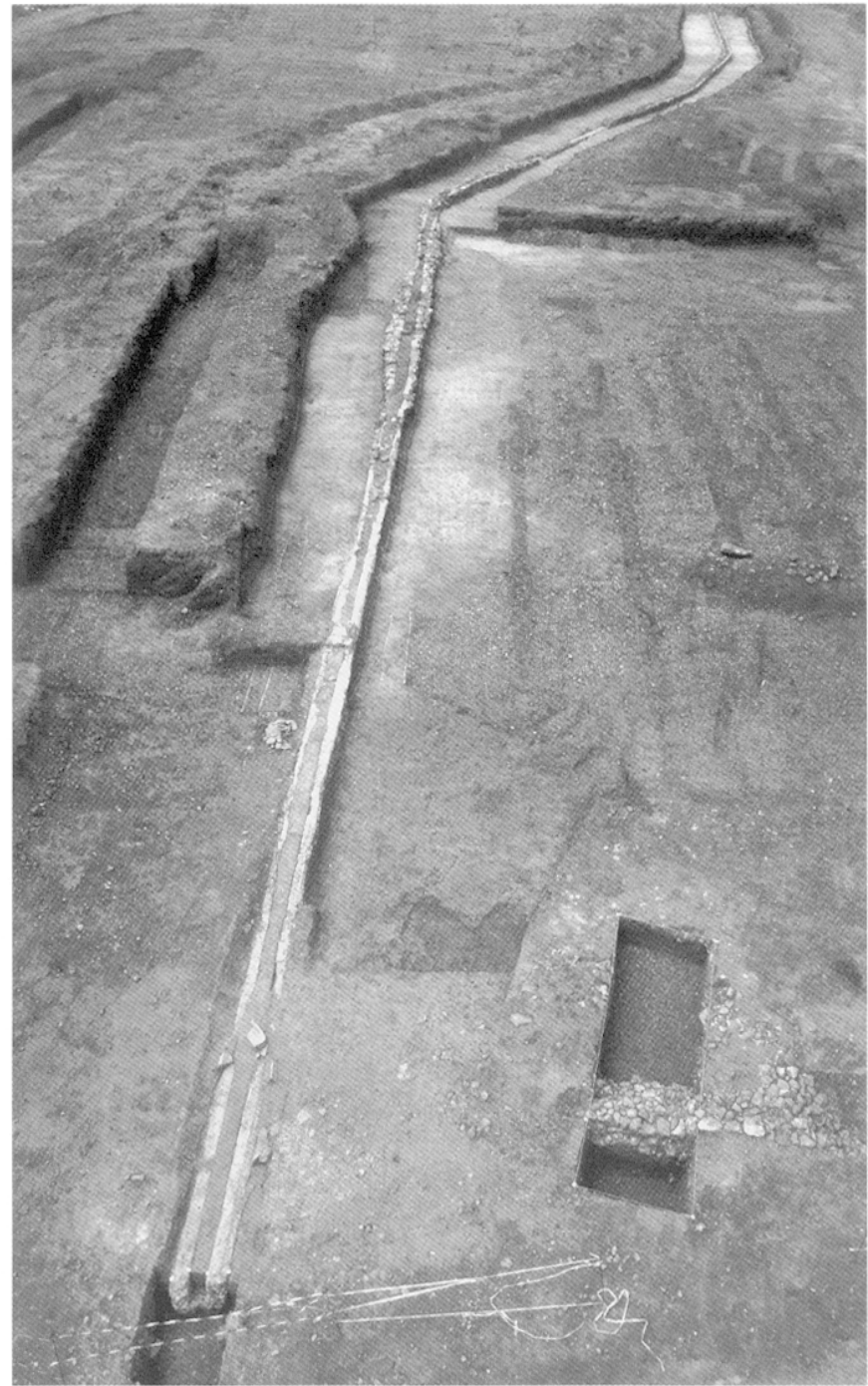

Fig. 130 - Villa de Vareilles : vue de l'aval des segments 4, 5, 6 et 7 de l'aqueduc haut en cours de fouille (cliché S. Mauné, CNRS).

canalisation qu'il s'est le mieux conservé, et son épaisseur varie de $4 \mathrm{~cm}$ à $9 \mathrm{~cm}$.

Dans un deuxième temps, les parois latérales de la conduite ont été surélevées de deux à trois assises sur une hauteur approximative de $0,25 \mathrm{~m}$. Le mortier utilisé est de moindre qualité que celui mis en œuvre pour le béton banché. De plus, cette surélévation n'a pas été observée sur toute la longueur de l'ouvrage

On doit insister sur le fait que de semblables observations ont été réalisées sur la totalité du parcours de l'aqueduc. Enfin, la partie supérieure des murets latéraux est, sur tout le parcours, largement chanfreinée. Toutes les sections relevées et dessinées amènent à des conclusions identiques : il s'agit d'une conduite homogène relevant d'un seul et même projet. Pourtant l'ouvrage n'est pas techniquement parfait; nous avons pu constater que se posait en particulier un problème de pente (fig. 133). En effet, entre le niveau du fond du canal relevé au point le plus en amont de la canalisation ct la cote altimétrique du même point relevé $222,50 \mathrm{~m}$ plus en aval, nous 


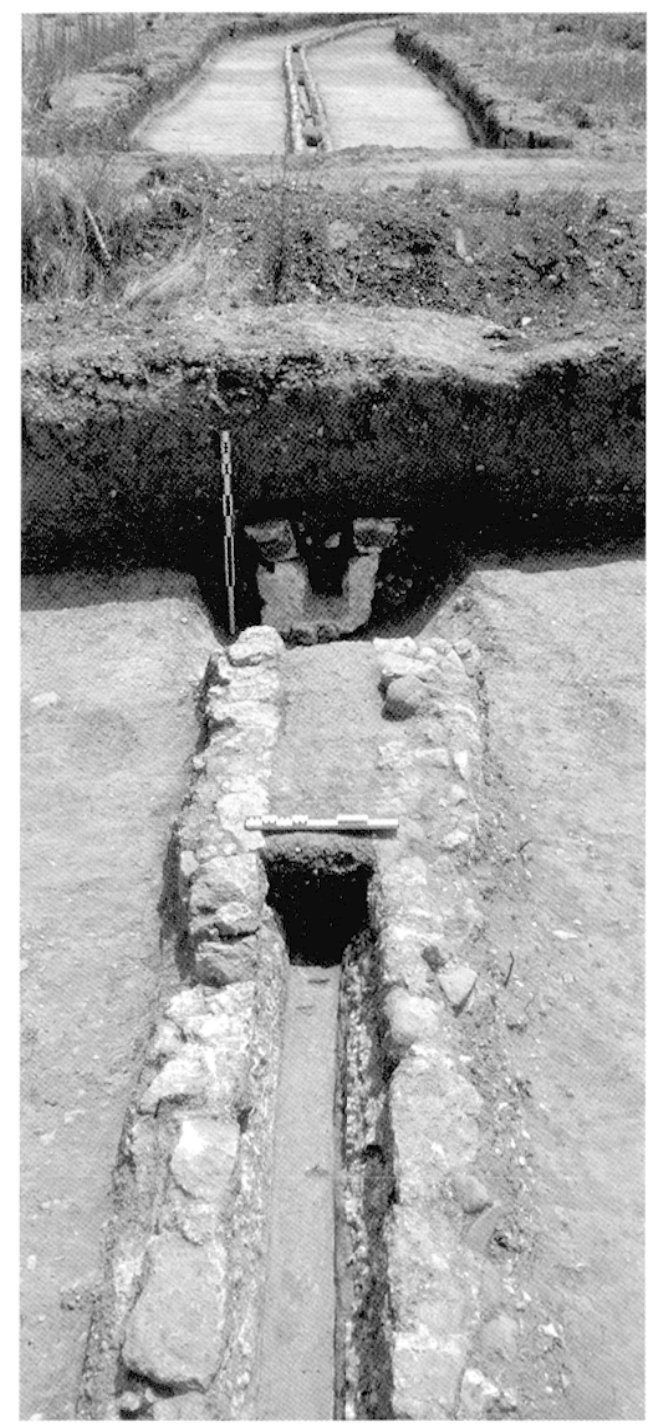

Fig. 131 - Villa de Vareilles : vue de l'amont d'une partie du segment 4 de l'aqueduc haut en cours de fouille (clichéS. Mauné, CNRS).

avons observé une contre-pente de $14 \mathrm{~cm}$. Cette valeur est évidemment incompatible avec la hauteur réduite de la conduite $(0,25 \mathrm{~cm}$ en moyenne). Pour remédier à cctte malfaçon de nivellement, le canal a été surélevé de une à deux assises afin d'empêcher tout débordement. Cette surélévation a été exclusivement mise en ceuvre aux endroits où le fond du canal était le plus bas. Elle est constituée de moellons de calcaire grossièrement équarris et de provenance différente, associés à des fragments de terres cuites architecturales et liés au mortier de chaux.

Concernant la question de la couverture éventuelle de cette conduite, trois hypothèses peuvent être formulćes.

La solution la plus simple consiste à supposer que la canalisation était obturée par des dalles de pierrc posées à plat. On pourrait aussi admettre que ces dalles ont été récupérées après l'abandon de la conduite et avant son recouvrement sous les épaisses couches de colluvionnement, mais la fouille n'a pas
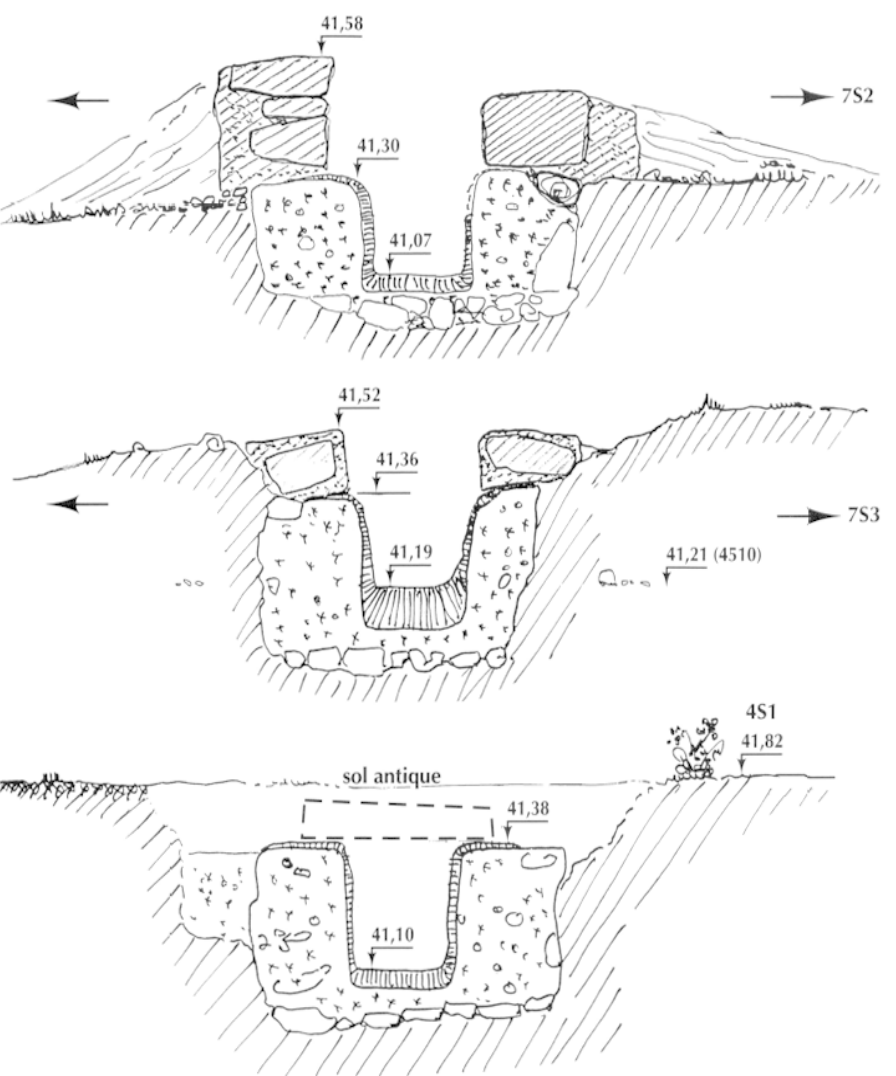

Fig. 132 - Villa de Vareilles : trois coupes de l'aqueduc haut montrant le rehaussement du canal dans certains segments (relevés 7. Sekhari et J.-L. Paillet, CNRS).

permis d'identifier la moindre trace de pose de dalles ni de leur spoliation dans ce secteur-là. La présence de quelques dalles de couverture à proximité immédiate de l'angle occidental du complexe viticole à cour centrale pourrait s'expliquer par l'importance de la fréquentation aux abords immédiats de la villa (passage dallé).

Une voûte pouvait couvrir la conduite. Mais, si cette voute avait existé, sa spoliation aurait laissé quelques traces sur la face supérieure qui n'ont pas été identifiées au moment de la fouille.

La canalisation était à ciel ouvert. C'est la solution originale sur laquelle nous nous arrêterons dans la mesure où la morphologie chanfreinée des murets de la canalisation semble confirmer cette hypothèse. De plus, si l'on prend en compte la nature de l'utilisation de l'eau transportée par cet aqueduc - qu'il faut indubitablement associer aux installations vinicoles et qui n'était donc pas destinée à l'habitation -, il est tout à fait envisageable de concevoir un conduit à l'air libre.

À partir des chiffres dont nous disposons et toujours selon la formule de Bazin, le débit théorique de ce second ouvrage est pour le premier état: $0,03536 \mathrm{~m}^{3} / \mathrm{s}$, soit $35 \mathrm{l} / \mathrm{s}$, soit $3055 \mathrm{~m}^{3} /$ jour et pour le second état : $0,06561 \mathrm{~m}^{3} / \mathrm{s}$, soit $65 \mathrm{l} / \mathrm{s}$, soit $5668 \mathrm{~m}^{3} /$ jour. 


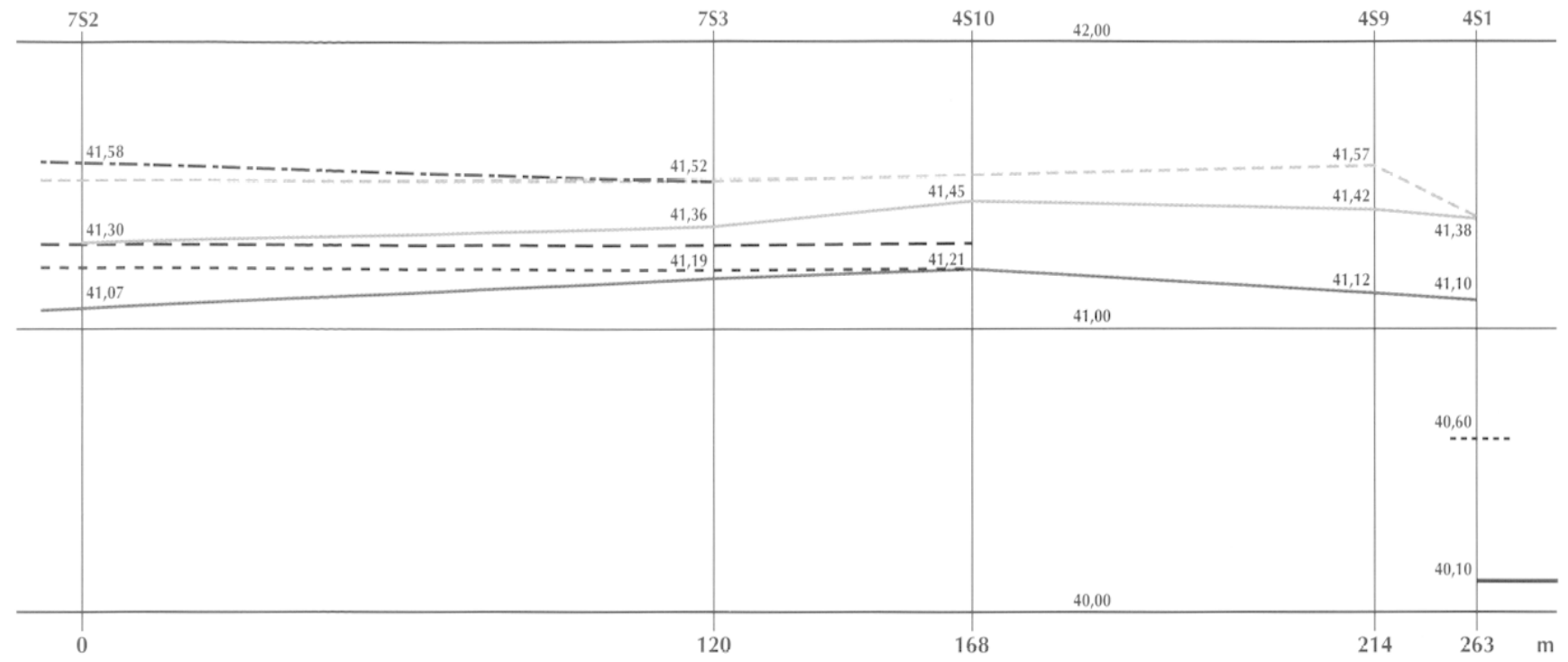

Fig. 133 - Villa de Vareilles : profil en long de l'aqueduc haut (J.-L. Paillet, CNRS).

\section{DATATION}

La position topographique du dernier segment - mais aussi celle de la fosse de spoliation de son réceptacle - et sa proximité immédiate avec l'angle occidental du complexe en $\mathrm{U}$ signalent à l'évidence un lien fort entre la structure hydraulique et les installations vinicoles, construites dans les années 40/50 apr. J.-C. Ce terminus post quem est confirmé par la position même de l'ouvrage au sein du parcellaire augustéen et par son installation dans le " paléosol » qui fonctionnait avec les vignes installées dans ce secteur. La fouille exhaustive des sédiments plaqués contre les parois externes de la conduite et résultant du rebouchage de la tranchée de fondation n'a fourni que très peu d'éléments de datation: une fibule presque complète datée par $M$. Feugère dans un large $I^{\mathrm{er}} \mathrm{s}$. apr. J.-C., ainsi qu'un bord de coupe Drag. 29b en sigillée sud-gauloise dont on situe habituellement l'apparition dans les années 40 apr. J.-C. C'est en fait la fouille d'un niveau de circulation lié à la construction de l'aqueduc qui nous permet d'asseoir une datation claudienne puisque le mobilier recueilli, certes peu abondant, regroupe notamment les restes attribuables à une dizaine de formes en sigillée, antérieures aux années 60/70 apr. J.-C. ${ }^{103}$. Ces observations nous paraissent donc concordantes pour placer la construction de l'aqueduc dans les années 40/50 apr. J.-C. Les réfections observées peuvent être immédiatement ultérieures à la mise en service de l'ouvrage ; elles peuvent aussi être imputées à la période qui voit l'apparition de trois nouveaux chais, durant la phase $V$.

103. Formes Drag. 29b, Drag. 18 et 19, Ritt. 12, Ritt. 8. On notera labsence révélatrice des formes Drag. $35 / 36$ et 37 , dont on situe l'apparition à l'extrême fin des années 60 apr. J.C.

\section{LA QUESTION DU CAPTAGE}

L'origine de l'eau qui alimentait les aqueducs de Vareilles n'a pas pu encore être déterminée avec précision. Les zones aquifères qui auraient pu être, dans l'Antiquité, mises à contribution sont situées assez loin de l'emprise de l'autoroute $\mathrm{A} 75$. Toutefois, nous avons effectué des prospections et des recherches dans la partie amont du bassin versant de Vareilles et, en fonction de la topographie, de l'obligation d'écoulement gravitaire de l'eau dans les aqueducs et de la situation des zones aquifères, nous avons travaillé sur trois hypothèses différentes.

La première privilégiait la présence d'un éventuel barrage construit situé en travers du lit du petit cours d'eau ${ }^{104}$. Rien dans la topographie du fond du thalweg, ni aucun vestige éventuel de semelle en béton de tuileau que n'aurait pas manqué de laisser un tel ouvrage ne permet de corroborer cette proposition ${ }^{105}$. De même, si un captage établi sur une retenue de ce type peut être envisagé pour l'irrigation ou l'alimentation des communs de la villa, cette solution semble improbable pour la distribution d'eau dans la pars urbana. Pour ce type d'usage, on préférait l'eau prélevée directement sur une source.

104. C'est à ce type de dispositif que pourrait renvoyer, selon I. Bermond, la présence dans le lit de la Vène (Balaruc-le-Vicux) d'un puissant massif de béton de tuileau mis au jour lors du curage de cette rivière (Lugand, Bermond, 2001, p. 98 et p. 205-206, fig. 183).

105. Il faut toutefois signaler qu'à environ $250 \mathrm{~m}$ de l'extrémité amont de l'aqueduc bas se trouve un chemin vicinal taluté qui est perpendiculaire à la rivière Vareilles et permet l'accès à un petit gué. Il faudrait pouvoir effectuer une coupe de cet aménagement pour s'assurer qu'il ne recouvre pas une structure bâtie plus ancienne. 
La seconde hypothèse découle de la présence, sur la rive gauche du Vareilles, à moins de $1 \mathrm{~km}$ au nord-ouest de la villa, du toponyme la Resclauze/Rasclause qui, dans cette région, désigne des aménagements bâtis liés à des sources importantes. L'exemple le plus emblématique est celui de la source de l'aqueduc de Béziers à Gabian (Andrieu, 1990), ou encore celui du dispositif de retenue associé à la source qui alimentait les moulins étagés de Neffiès, dans l'emprise du site antique et médiéval de Saint-Étienne-de-Trignan, situé à une quinzaine de kilomètres au sud-ouest de Vareilles (Mauné, 1998, p. 407-408). Dans ces deux cas, la source est désignée localement sous le terme de Resclause/Resclauze. Actuellement, la végétation très dense qui se trouve dans ce secteur de rebord de plateau ne permet pas d'investigations approfondies. Soulignons toutefois que l'eau provenant de cette zone aurait dû emprunter pour rejoindre la villa un petit ouvrage d'art (un ponceau) traversant le Vareilles, ouvrage dont nous n'avons aucune trace sur le terrain.

Une dernière hypothèse a été examinée, celle de la présence d'une source pérenne située au point de départ du bassin versant où la tradition orale villageoise (à Adissan) situe une gloriette - un regard bâti - à partir de laquelle le village était alimenté en eau potable au XIX ${ }^{\mathrm{e}} \mathrm{s}$. ${ }^{106}$. L'emplacement de ce captage est aujourd'hui totalement oublié, mais il alimente encore un grand bassin situé non loin de la cave coopérative. $\mathrm{Si}$ cette source s'avérait être celle utilisée par les occupants de la villa, la distance couverte par les aqueducs serait d'environ $2 \mathrm{~km}$.

Quoi qu'il en soit, ces hypothèses ne nous paraissent pas contradictoires car il y a deux aqueducs à Vareilles et leurs usages semblent distincts. Nous ignorons en effet s'il existait un raptage commun trouvant son origine au point de départ de l'ouvrage augustéen, ou bien si chacun des deux aqueducs plus récents captait l'eau en deux endroits différents. Même si la fouille indique que le tronçon dégagé de l'aqueduc haut semble - lorsque l'on prolonge son tracé vers l'amont provenir du même secteur que l'aqueduc bas, il faudra attendre la poursuite des investigations archéologiques pour préciser cet aspect de la problématique.

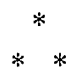

La mise en corrélation des estimations de débit maximales qui viennent d'être présentées, dans une perspective chronologique, nous conduit à considérer que dans un premier temps la villa augusto-tibérienne étair abondamment alimentée en eau, avec un débit potentiel maximal de $6500 \mathrm{~m}^{3}$. Les années 40 apr. J.-C. marquent vraisemblablement une rupture dans l'évolution du site et de son alimentation en eau. En effet, le réservoir qui recevait et distribuait l'eau ainsi que le dernier segment de l'ouvrage sont détruits par la construction du grand bâtiment à plan en U. Cet événement semble marquer l'abandon pur et simple de l'ourrage qui devait présenter, quelques décennies après sa construction, des signes de faiblesse. Peut-être reconstruit-on, sur la plus grande partie de son tracé, un aqueduc en béton de tuileau, mais nous avons soulevé plus haut les incertitudes chronologiques qui pèsent

106. Nos remerciements vont au maire d'Adissan, M. Imbert, qui a bien voulu nous renseigner sur cette question. sur cette proposition. Le nouveau bâtiment, de grande ampleur, arait besoin d'eau et pour l'alimenter le premier aqueduc haut est établi sur les terrasses supérieures. Cet ouvrage qui devait pourvoir à suffisance le nouvel établissement ne lui apporte cependant que $3000 \mathrm{~m}^{3}$ d'eau par jour, soit globalement deux fois moins. Une contre-pente involontaire de son radier explique la faiblesse de ce débit. C'est peut-être dans les années 70 apr. J.-C., lorsqu'un projet de construction de bâtiment thermal et de communs est établi, que l'on reprend le tracé de l'aqueduc bas et que l'on reconstruit une conduite pour alimenter l'ensemble des nouveaux bâtiments et peut être aussi un second moulin (moulin ouest). Désormais, on est certain que le volume d'eau journalier disponible est égal à la somme des débits des deux ouvrages en service, 3000 + 2500 litres, soit $5500 \mathrm{~m}^{3}$. À ce moment-là ou ultérieurement (phase V), la hauteur du canal de l'aqueduc haut est augmentée de manière à accroître son débit. On dépasse dans ces conditions $8700 \mathrm{~m}^{3}$ d'eau par jour. Que représentent ces chiffres? Rappelons ici qu'il s'agit de débits potentiels maximaux, mais qu'en réalité nous ignorons la hauteur du fil d'eau dans les différents aqueducs. En revanche, ce qui pour nous est d'une extrême importance c'est la relation de proportionnalité entre les différents débits qui, en tout état de cause, restera toujours semblable. D'autre part, ces volumes d'eau quotidiens présentent aussi l'intérêt de pouvoir être comparés avec ceux des aqueducs urbains. Il faut en conclure que ces aqueducs domaniaux avaient un débit six à dix fois inférieurs à des ouvrages comme les aqueducs d'Arles et de Nîmes qui alimentaient des cités où vivait une population nombreuse. En conséquence, il faut bien admettre que ces ouvrages ruraux étaient surdimensionnés par rapport aux besoins réels des 100 ou 150 personnes résidant ou travaillant à Vareilles. Le souci d'éviter que ces aqueducs ne se bouchent explique sans doute ce surdimensionnement. Quoi qu'il en soit, ces énormes quantités d'eau pouvaient également - au moins dans le cas de l'aqueduc haut - être utilisées pour l'irrigation. À proximité immédiate de l'emplacement du castellum de l'aqueduc haut, une série de fosses de plantation arbustive constitue peut-être le seul aménagement conservé de jardins vivriers dont nous ignorons l'extension réelle. L'apport d'une telle quantité d'eau à une partie des cultures domaniales pouvait constituer, en terme de rendements, un atout extrêmement intéressant dans une région coutumière d'étés secs et chauds.

D'un point de vue plus large, l'apport des fouilles préventives de Vareilles à la question de la petite hydraulique rurale est double: en premier lieu, il concerne les techniques de construction des aqueducs qui, depuis une source ou une rivière et sur des tracés relativement courts, approvisionnaient en eau courante des habitations et des installations agricoles.

L'ourrage le plus ancien est le moins bien conservé puisque ne subsiste qu'une partie de sa semelle de fondation : cette dernière nous permet cependant de proposer l'hypothèse de la présence d'une construction en grand appareil destinée à alimenter la villa d'époque julio-claudienne. L'analyse et l'étude des deux aqueducs plus récents indiquent en revanche qu'il s'agit de constructions relativement peu soignées, que l'on peut même qualifier de frustes : si elles ont nécessité un volume important de matériaux et notamment de béton hydraulique, il 
n'en demeure pas moins que techniquement de nombreuses erreurs ou approximations dans leur mise en œurre ont dû entraîner des problèmes auxquels il a fallu rapidement remédier. C'est surtout l'aqueduc haut qui permet de faire, à cet égard, les obserrations les plus intéressantes. Son concepteur s'est trompé lorsqu'il a réalisé le nivellement général et le profil en long de l'ouvrage qu'il a fallu par la suite rehausser. Il ne fait guère de doute que la main-d'œuvre employée lors de sa construction ne disposait pas de la même formation ni des mêmes connaissances que les ingénieurs œuvrant sur des ouvrages publics. Toutefois, si ce constat semble valable pour tout ce qui concerne les aqueducs - encore qu'il nous faudrait connaître pour être certain de notre jugement, les installations de captages -, il l'est moins pour les aménagements hydrauliques de la villa et notamment les moulins construits à l'extrémité du dispositif. Ceux-ci témoignent en effet d'une technicité évoluée et nous orientent vers une sphère plus spécialisée (Mauné, Paillet, 2003).

Le second point sur lequel nous voudrions insister est celui de la place de l'hydraulique dans les grands centres domaniaux du Haut-Empire.

Ce qui frappe en effet quand on regarde le plan de la villa de Vareilles, c'est l'importance de l'eau et des aqueducs, et l'absence de relation avérée avec la petite rivière toute proche, si ce n'est pour y déverser les eaux usées et industrielles. Il y a peut-être là une indication sur le caractère intermittent de ce cours d'eau dans l'Antiquité : les propriétaires de la villa ont pu $s$ 'affranchir de cette contrainte en allant chercher l'eau bien en amont, probablement à une ou plusieurs sources pérennes.

L'utilisation de l'eau à Vareilles était multiple et variée. On voit bien notamment que l'aqueduc haut était avant tout destiné aux installations vinicoles; la relation topographique entre le point d'arrivée de cet ouvrage et l'angle sud-ouest du grand complexe en $\mathrm{U}$ paraît évidente, comme la nécessité de pouvoir nettoyer les salles de foulage et de presse ainsi que les 350 dolia du grand chai en L (Mauné, 2003a).

Lorsque l'eau n'était pas utilisée pour les installations vinicoles, peut-être était-elle dirigée vers la pars urbana, mais on ne peut exclure un usage d'irrigation pour des cultures vivrières installées le long de l'aile sud du grand complexe en $U$ par exemple. La présence de trois moulins hydrauliques indique aussi que l'on a tiré parti de la maîtrise de l'eau pour produire, à moindre frais - si ce n'est l'investissement de départ -, de grandes quantités de farine, utilisée pour nourrir la population, sans doute nombreuse, du domaine.

Il reste qu'il est difficile d'apprécier, même de façon globale, les besoins en eau d'une villa de ce type car les ouvrages de stockage et de distribution ont été totalement épierrés ou détruits. Hommes, bêtes et installations artisanales diverses ne devaient pas nécessiter d'énormes volumes - seulement un flux constant et régulier - mais il fallait en revanche pouvoir, durant des laps de temps assez courts, répondre à des besoins spécifiques très importants : époque des vendanges ou périodes d'utilisation des thermes par exemple. La présence de deux aqueducs avant fonctionné de façon plus ou moins contemporaine semble avoir été la réponse la plus appropriée pour répondre à cette nécessité, durant la période d'activité maximale de la villa (phases IV et V).

Quoi qu'il en soit, le plan de la villa fut, au moins à partir de la phase III, dicté par des impératifs liés à la maîtrise effective de l'eau, tant en ce qui concerne les questions d'approvisionnement que celles relevant de l'évacuation. À cet égard, l'analyse topographique de la position et des directions empruntées par les égouts permet de s'interroger sur la présence, hors de l'emprise de la fouille, d'autres bâtiments situés immédiatement à l'est des vestiges appréhendés en 2002. De même le tracé des aqueducs, avant leur jonction avec les bâtiments de la villa, peut, dans ce cas comme dans d'autres, nous informer sur l'extension du fundus car il est très probable que de tels ouvrages empruntaient un cheminement situé dans l'emprise de la propriété ${ }^{107}$. À cet égard, on peut qualifier les aqueducs ruraux de véritables épines dorsales domaniales pouvant également servir de limite parcellaire et structurer la desserte des champs. L'étude de leur tracé précis ouvre donc, au-delà des seules questions liées à la petite hydraulique rurale, bien des perspectives de recherche.

107. Pour J.-G. Gorges, que nous avons consulté sur cette question et qui a cu l'amabilité de nous répondre précisément sur ce sujet, " il s'agit là d'une précaution élémentaire et logique, compte tenu du fait que tous les contentieux sur l'eau connus par exemple en Espagne portent justement sur des détournements d'eau à partir d'aqueducs publics (par ex. Blazquez, 1977). L.es propriétaires privés avaient intérêt à conserver toute la maîtrise de leur bien, et la taille des domaines de ceux qui disposaient de telles installations leur permettait certainement de répondre à cette nécessité". 